\title{
Dynamic modelling of retrogressive landslides with emphasis on the role of clay sensitivity
}

\author{
Xue Zhang ${ }^{1,2}$ | Scott W. Sloan ${ }^{3}$ | Eugenio Oñate ${ }^{2}$
}

\author{
${ }^{1}$ Department of Civil Engineering and \\ Industrial Design, School of Engineering, \\ University of Liverpool, Liverpool, UK \\ ${ }^{2}$ International Centre for Numerical \\ Methods in Engineering (CIMNE), \\ Barcelona, Spain \\ ${ }^{3}$ ARC Centre of Excellence for \\ Geotechnical Science and Engineering, \\ University of Newcastle, Callaghan, \\ Australia
}

\section{Correspondence}

Xue Zhang, Department of Civil Engineering and Industrial Design, School of Engineering, University of Liverpool, Liverpool, UK.

Email: xue.zhang2@liverpool.ac.uk

Funding information

Marie Skłodowska-Curie Actions Individual Fellowship, Grant/Award Number: 744281; Australian Research Council Centre of Excellence for Geotechnical Science and Engineering

\begin{abstract}
Summary
This paper presents a detailed numerical study of the retrogressive failure of landslides in sensitive clays. The dynamic modelling of the landslides is carried out using a novel continuum approach, the particle finite element method, complemented with an elastoviscoplastic constitutive model. The multiwedge failure mode in the collapse is captured successfully, and the multiple retrogressive failures that have been widely observed in landslides in sensitive clays are reproduced with the failure mechanism, the kinematics, and the deposition being discussed in detail. Special attention has been paid to the role of the clay sensitivity on each retrogressive failure as well as on the final retrogression distance and the final run-out distance via parametric studies. Moreover, the effects of the viscosity of sensitive clays on the failure are also investigated for different clay sensitivities.
\end{abstract}

\section{KEYWORDS}

landslides, PFEM, retrogressive failure, sensitive clay, strain softening

\section{INTRODUCTION}

Landslides in sensitive clays have long been recognised as a challenging problem. Investigations show that, if sensitive clay is present, a seemingly stable area might be subject to a major landslide after a small initial failure. Typical examples of these landslides occurred in Canada, ${ }^{1}$ where they proceeded retrogressively up to even kilometres away from the place they were initiated.

To minimise the degree of destruction that these landslides can cause, a better understanding of their behaviour in sensitive clays is of great importance. Many efforts have so far been made in this respect, for instance through theoretical analysis, ${ }^{2-5}$ experimental tests, ${ }^{6-10}$ and empirical statistics. ${ }^{11-14}$ In these studies, special emphasis was typically given to the triggering mechanisms, the evolution processes, and the retrogression run-out distances. Despite these contributions, landslides in sensitive clays are not yet fully understood. Empirical formulations and analytical solutions indeed provide valuable information; they generally involve simplified assumptions, and the information they yield is limited. Laboratory tests play an important role in understanding landslides. Nonetheless, they are in many cases of high cost as well as of long duration for model construction, especially for centrifuge testing. For these reasons, laboratory tests usually focus on only a limited number of variables. As an alternative, computational modelling can be adopted.

Although detailed parametric studies can be performed in virtual experiments, the numerical simulation of landslides in sensitive clays is never a trivial task. The conventional limit equilibrium method and/or the finite element 
strength reduction method are the commonly used approaches for slope stability analysis in engineering practice. Neither of them performs well when sensitive clays are involved. Indeed, both these methods may assign a factor of safety above 1 to a slope which actually is unstable because of their assumption of a perfectly plastic soil model. It is known that landslides in sensitive clays develop in a progressive manner because of the strength reduction of soils. ${ }^{15}$ If for any reason (eg, earthquakes, erosion, or human activity) the stress state of a point within a slope moves past its peak strength, the strength at that point may reduce significantly with further deformation. The unbalanced stress is, consequently, transferred to its surrounding material whose peak strength may also be exceeded. The unbalanced stresses continue to migrate in such a manner until a critical failure surface is formed in the slope. This particular type of progressive failure process requires a complete deformation analysis with the strain-softening behaviour of the material being accounted for. ${ }^{16}$ However, it is worth noting that the inclusion of the strain softening may result in the solution becoming strongly mesh dependent. This stems from the fact that the related boundary value problem is no longer elliptic in statics or hyperbolic in dynamics as indicated by Belytschko and Lasry. ${ }^{17}$ To overcome this issue, special regularisation techniques, such as nonlocal theories or the introduction of artificial viscosity, have to be adopted.

The numerical modelling of the postfailure stage of landslides in sensitive clays is even more complex. For example, in multiple retrogressive earthflows, materials from the initial slide flow out of the generated crater at the front of the deposit leading to a new backscarp which may be unstable and collapse as well. Clays involved in this second failure also migrate away resulting in another new backscarp. Such retrogressive collapse continues until a final stable scarp forms. The state of sensitive clays, in particular, those of high sensitivity (eg, quick clays), may evolve from a solid to a viscous liquid in this process. The transition from a solid-like behaviour to a fluid-like behaviour inevitably leads to extreme material deformation and rapidly changing evolutions of free surfaces. Computational modelling of the postfailure stage of landslides in a sensitive clay thus requires not only an appropriate constitutive model to describe both the solid-like and the liquid-like behaviours of the involved material but also a robust numerical technique that is capable of tackling dramatic changes in the geometry. A recent contribution in this regard was made by Wang

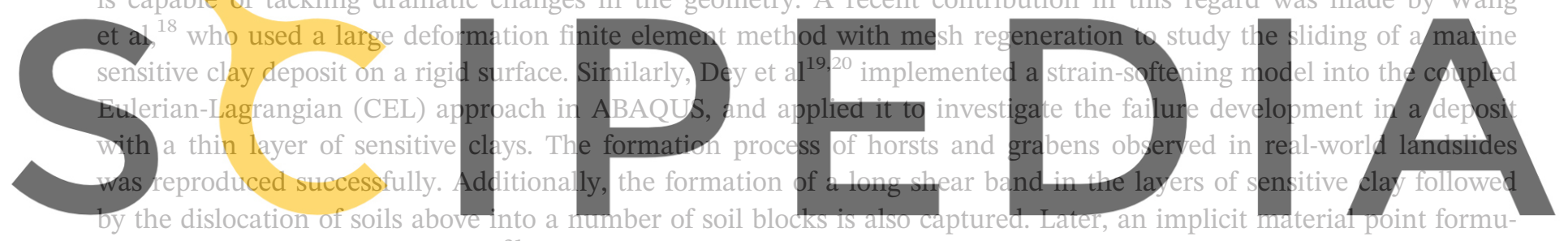

lation was proposed in Wang et $\mathrm{al}^{21}$ for modelling the retrogressive failure of a sensitive clay slope. Note that, although

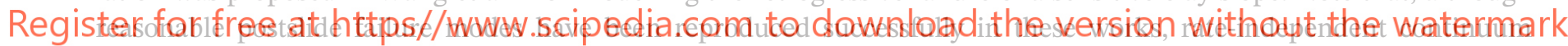

models were adopted. The neglect of the rheological property of sensitive clays, for example the viscosity, has an adverse effect on the correct prediction of landslides in sensitive clays, ${ }^{22-25}$ particularly for clays of high sensitivity that behave more like liquids after they are fully remoulded. More recently, a Lagrangian formulation of elastoviscoplasticity was proposed in Zhang et $\mathrm{al}^{26}$ for analysing large deformation problems related to sensitive clays. In the formulation, the elastoviscoplastic model, which is a mixture of Tresca model with strain softening and the classical Bingham model, was used to describe the complex behaviour of sensitive clays. To tackle the issues related to the extreme change of geometry, for example mesh distortion and free-surface evolutions, the model was implemented into the framework of the particle finite element method. ${ }^{27,28}$ It has been shown in Zhang et $\mathrm{al}^{26}$ that this approach is capable of simulating progressive failure of geostructures in sensitive clays with a typical example being the collapse of a column of sensitive clay. Moreover, its effectiveness and robustness in simulating landslides in sensitive clays have been illustrated as well in Zhang et al. ${ }^{26}$

Although the landslide simulation was carried out in Zhang et al, ${ }^{26}$ it served as a general example to show the capability of the numerical scheme and the constitutive model. In this study, the computational framework proposed in Zhang et $\mathrm{al}^{26}$ is adopted for a detailed numerical investigation of the retrogressive landslide in sensitive clays with emphases on the mechanism of the multiple retrogressive failure mode, the kinematics of the sliding mass, and the resulting deposition (eg, the final run-out distance and the retrogression distance). Special attention is devoted to studying the role of the sensitivity of clays on the failure, including its effects on the final retrogression and run-out distance as well as on each new retrogressive collapse, via parametric studies. Last but not least, the focus is also placed on the effect of the viscosity of clays on the progressively retrogressive failure, in particular, its influence on the final retrogression distance and the final run-out distance when the clays are of different sensitive degrees.

The paper is organised as follows. Section 2 presents the problem considered and the corresponding governing equations for dynamic modelling. The material parameters utilised in the numerical simulation are summarised in Section 3 , 
and the particle finite element method is described briefly in Section 4. Section 5 then provides the simulation results and discussions before conclusions are drawn in Section 6.

\section{PROBLEM DESCRIPTION}

To investigate landslides in sensitive clays, the problem illustrated in Figure 1 is studied. The deposit involves a slope of height $H$ and length $2 H$. The extent of the deposit is chosen to be sufficiently long so that there is no effect from the fixed boundary on the left. For simplicity, the problem is regarded as plane strain.

Suppose the volume of the deposit is represented by $\Omega$ and the surface is denoted by $\Gamma$. The partition of the surface is $\Gamma=\Gamma_{\mathrm{u}} \cup \Gamma_{\mathrm{t}}$, where $\Gamma_{\mathrm{u}}$ and $\Gamma_{\mathrm{t}}$ are the kinematic and traction boundaries obeying the constraint $\Gamma_{\mathrm{u}} \cap \Gamma_{\mathrm{t}}=\varnothing$ with $\varnothing$ being a null set. The momentum conservation equations, the kinematic equations for the displacement gradients, and the corresponding boundary conditions then read

$$
\begin{aligned}
& \nabla^{\mathrm{T}} \boldsymbol{\sigma}+\mathbf{b}=\rho \ddot{\mathbf{u}} \quad \text { in } \Omega \\
& \varepsilon=\nabla^{\mathrm{T}} \mathbf{u} \quad \text { in } \Omega \\
& \mathbf{u} . \overline{\mathrm{u}} \quad \text { on } \Gamma_{\mathrm{u}} \\
& \mathbb{N}^{\mathrm{T}} \sigma=\bar{t} \text { on } \Gamma_{\mathrm{t}}
\end{aligned}
$$
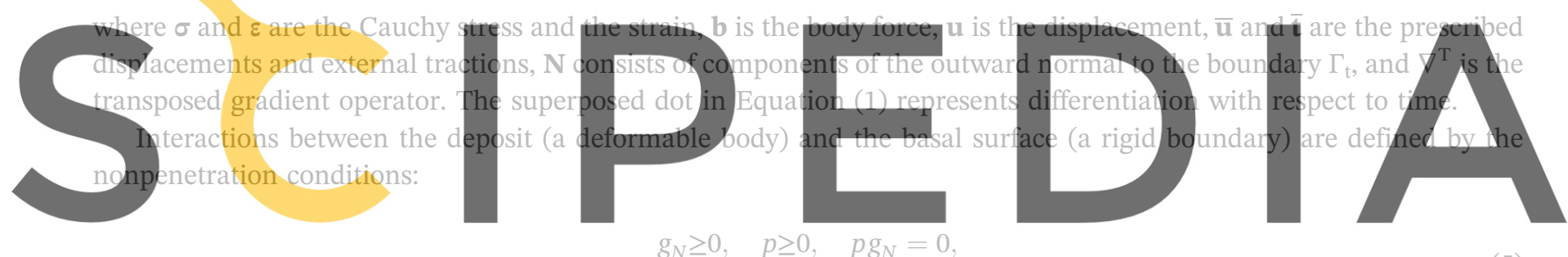

$g_{N} \geq 0, \quad p \geq 0, \quad p g_{N}=0$

Register for free at https//www.scipedialcomite download the version without the watermark

where friction is also taken into account. In the above, $g_{N}$ is the gap between the material and the rigid surface, $p$ is the contact pressure which is positive corresponding to compression, $q$ is the tangential stress, and $\mu_{F}$ is the friction coefficient between the material and the surface. The above conditions are imposed on all boundary material points of the deposit. After finite element discretisation, they are imposed on the boundary nodes of the mesh. More details of these nonpenetration conditions can be found in Zhang et al. ${ }^{29}$ Notably, the rigid surface is usually assumed to be rough for considering its interaction with clays ${ }^{19}$; it is however not uncommon to make an assumption of a friction contact mechanism between the clay and the rigid base when reproducing real-world landslides in clays. ${ }^{30}$ In this study, the contact between the clay and the basal surface is assumed to be frictional following Llano-Serna et al. ${ }^{30}$

The behaviour of the sensitive clay is described by an elastoviscoplastic model with strain softening. If the stress state is inside the yield domain, namely the yield function $F(\sigma, \kappa)<0$ where $\kappa$ is an internal variable for strain softening, the deformation is elastic. The total strain rate $\dot{\varepsilon}$ is then the elastic strain rate $\dot{\varepsilon}^{\mathrm{e}}$ calculated via Hooke's law as

$$
\dot{\varepsilon}^{\mathrm{e}}=\mathbb{C} \dot{\sigma}
$$

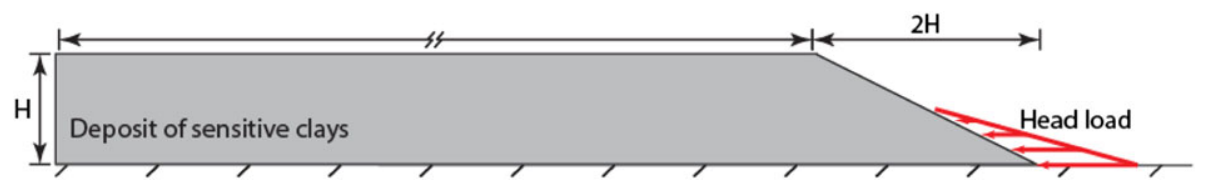

FIGURE 1 Schematic diagram for a deposit of sensitive clays [Colour figure can be viewed at wileyonlinelibrary.com] 
where $\mathbb{C}$ is the elastic compliance matrix. Otherwise, an additive decomposition applies to $\dot{\varepsilon}$ according to

$$
\dot{\varepsilon}=\dot{\varepsilon}^{\mathrm{e}}+\dot{\boldsymbol{\varepsilon}}^{\mathrm{vp}}
$$

where $\dot{\varepsilon}^{\mathrm{vp}}$ is the viscoplastic strain rate. It has been shown in Locat and Demers ${ }^{31}$ that the classical Bingham model captures the rheological behaviour of Canadian sensitive clays well, and is thus adopted in this work. The total stress is

$$
\sigma=\tau+\eta \dot{\varepsilon}^{\mathrm{vp}}
$$

where $\eta$ is the viscosity coefficient, $\tau$ is the stress lying on the boundary of $F(\tau, \kappa)=0$, and the quantity $\sigma-\tau$ is called the overstress. The viscoplastic strain rate is taken to be normal to the yield surface at $\tau$ :

$$
\dot{\varepsilon}^{\mathrm{vp}}=\dot{\lambda} \nabla_{\tau} F(\tau, \kappa)
$$

where $\dot{\lambda}$ is the non-negative plastic multiplier rate and $\nabla_{\tau}(*)$ denotes the derivative of $(*)$ with respect to $\tau$. In the limiting case of $\eta=0$, the elastoviscoplastic model reduces to the classical rate-independent elastoplastic model.

The Tresca yield criterion used here may be expressed as

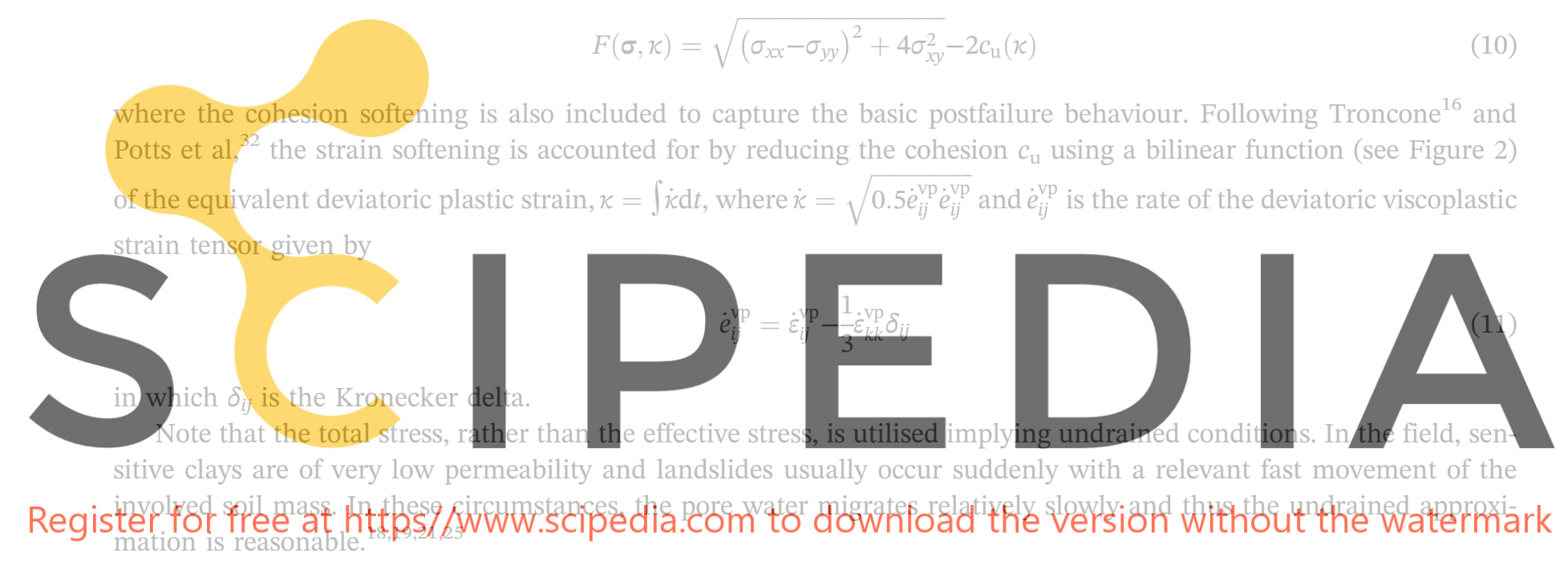

\section{SELECTION OF MATERIAL PARAMETERS}

Sensitive clays are commonly classified based on their sensitivity, St, which is defined as the ratio of their peak strength, $c_{\text {up }}$, to their remoulded strength, $c_{\text {ur }}$ (see also Figure 2). Most natural clays are sensitive except for heavily overconsolidated and boulder clays. Table 1 illustrates the classification of sensitive clays defined according to Skemption and Northey. ${ }^{33}$ Clays with $1<\mathrm{S}_{\mathrm{t}} \leq 2$ are regarded as having low sensitivity. Normally consolidated clays are usually of medium sensitivity $2<\mathrm{S}_{\mathrm{t}} \leq 4$, while highly sensitive clays of $4<\mathrm{S}_{\mathrm{t}} \leq 8$ are also encountered frequently in the field. Clays are considered to be extra-sensitive if $S_{t}>8$ and to be quick if $S_{t}>16$. To select appropriate material properties, the correlation between the remoulded undrained strength and the sensitivity of clays involved in real-world landslides that occurred in Canada ${ }^{11}$ and Norway ${ }^{2}$ are drawn in Figure 3 where the properties of sensitive clays in some

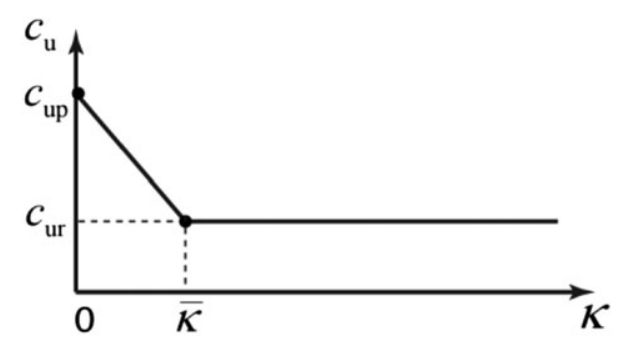

FIGURE 2 Variations of the undrained shear strength $c_{\mathrm{u}}$ with the equivalent deviatoric plastic strain represented by parameter $\kappa$ 
TABLE 1 Classification of sensitive clays according to Skemption and Northey ${ }^{33}$

\section{Classification}

Insensitive clays

Low-sensitive clays

Medium-sensitive clays

Highly sensitive clays ${ }^{\mathrm{a}}$

Extra-sensitive clays

Quick clays
St

$\sim 1$

$1-2$

$2-4$

$4-8$

$>8$

$>16$

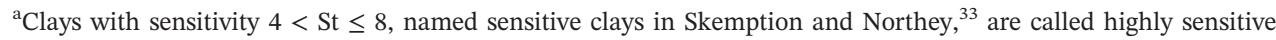
clays in this study for the convenience of description.
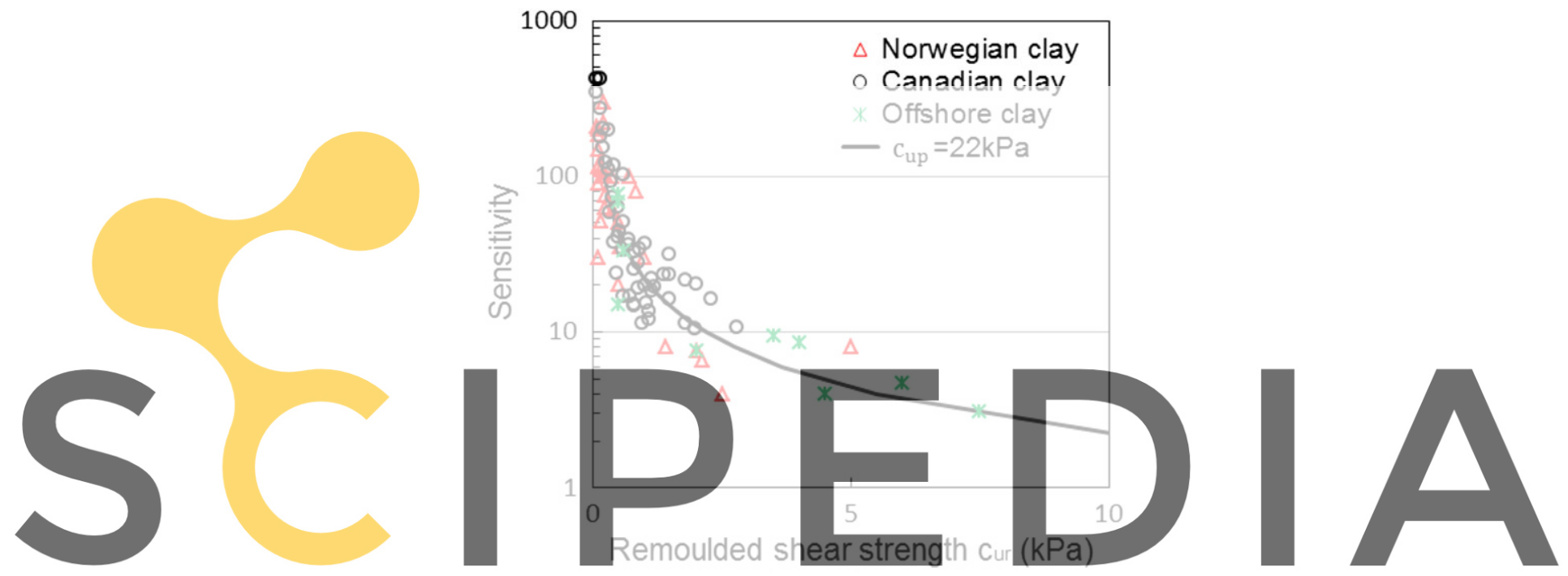

the sensitivity and the remoulded shear strength based on available data. ${ }^{2,11,34,35}$ The solid line

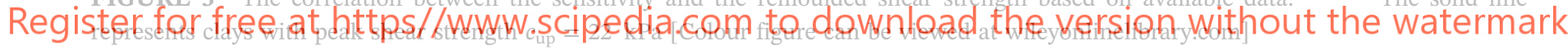

offshore sites 34,35 are also included. Because the geometry of the deposit is fixed in this study, the intact undrained shear strength is set to be $22 \mathrm{kPa}$ (corresponding to the solid line in Figure 3) so that the factor of safety of the initial slope is fixed in all cases. The sensitivity, however, varies in parametric studies carried out in Section 5.2 to cover the range of St given in Table 1. Notably, the undrained shear strength in practice usually increases with the depth; the value of $22 \mathrm{kPa}$ used in this study is assumed to be the average undrained shear strength of the deposit, which is a common approximation when modelling a landslide in clays. ${ }^{19,20}$

According to Quinn et al, ${ }^{5}$ the relation between the shear stiffness, $G$, and the peak shear strength, $c_{\text {up }}$, for undisturbed sensitive clays yields $\mathrm{G} / c_{\mathrm{up}} \cong 30-100$. Given that $c_{\text {up }}$ is assumed to be $22 \mathrm{kPa}$ and Poisson ratio is set to be

TABLE 2 Reference material properties of sensitive clays

\section{Material Parameters}

Young modulus $E$

Poisson ratio $v$

Unit weight $w$

Reference equivalent deviatoric plastic strain $\bar{\kappa}$

Peak cohesion $c_{\text {up }}$

Sensitivity St

Viscosity coefficient $\eta$

Friction coefficient $\mu_{F}$

\section{Value}

$4.26 \times 10^{6} \mathrm{~Pa}$

0.49

$20 \mathrm{kN} / \mathrm{m}^{3}$

0.6

$22 \mathrm{kPa}$

6

$500 \mathrm{~Pa} \mathrm{~s}$

0.36 
0.49 to approximate undrained (constant volume) conditions, after determining $G$, Young modulus $(E)$ can be calculated which ranges from $1.96 \times 10^{6} \mathrm{~Pa}$ to $6.55 \times 10^{6} \mathrm{~Pa}$. In all simulations, an average value of $E=4.26 \times 10^{6} \mathrm{~Pa}$ is adopted. The unit weight is assumed to be $w=20 \mathrm{kN} / \mathrm{m}^{3}$, which is typical for a sensitive clay, ${ }^{7}$ and the reference equivalent deviatoric plastic strain is $\bar{\kappa}=0.6$ according to Quinn et al. ${ }^{5}$ Back calculations of various subaerial and submarine slides in Edgers and Karlsrud ${ }^{23}$ and Johnson and Rodine ${ }^{24}$ suggested the value of viscosity ranging from 100 to $1499 \mathrm{~Pa}$ s, which is in line with the value used for reproducing the Storegga slide in a sensitive marine clay in Gauer et al. ${ }^{25}$ Thus, the viscosity of the clays in the reference analysis is assumed to be $500 \mathrm{~Pa} \mathrm{~s}$ which is within the suggested range. Its influence will also be investigated via parametric studies. The basal surface is assumed to have a friction coefficient of 0.36 . For the sake of convenience, the reference material parameters utilised in the simulation are listed in Table 2 .

\section{PARTiCle Finite ELEMENT MODELling}

The particle finite element method (PFEM) ${ }^{28,36,37}$ is adopted for the numerical simulation in this study. The PFEM is a mixture of the Lagrangian finite element method (FEM) and the particle approach. The simulation in a typical time interval $\left[t_{n}, t_{n+1}\right]$ proceeds by first solving the governing equations on the meshes ( $M_{n}$ as shown in Figure 4$)$ assigned to the computational domain using the Lagrangian FEM. After the mesh nodes are updated to their new positions according to the computed displacement increment, they are considered as free particles denoted by $C_{n}+1$ in Figure 4 . The new computational domain $\Omega_{n+1}$ is identified on the basis of $C_{n+1}$, using the so-called alpha-shape method, and then discretised using a new mesh. After mapping the state variables (such as velocities, accelerations, stresses, and strains) to the new mesh $M_{n+1}$, an incremental finite element analysis is conducted again and the simulation then steps into the next time interval. ${ }^{28}$ Because of its nature, the PFEM inherits not only the ability of a particle approach to handle general large deformation problems but the solid mathematical foundation of the conventional finity element method. To date, of a real-world flow-like land PFEM presented in Zhang et for elastoviscoplastic analysis tion algorithm. A major
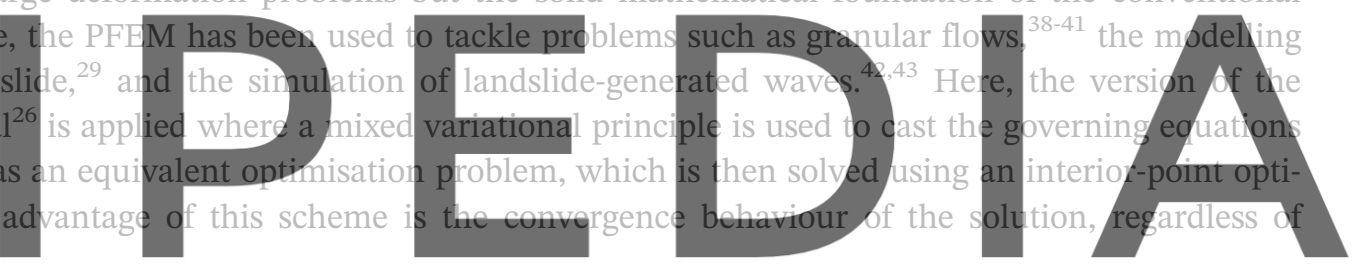

Register for free at https//www.scipedia.com to download the vestsion without the watermark
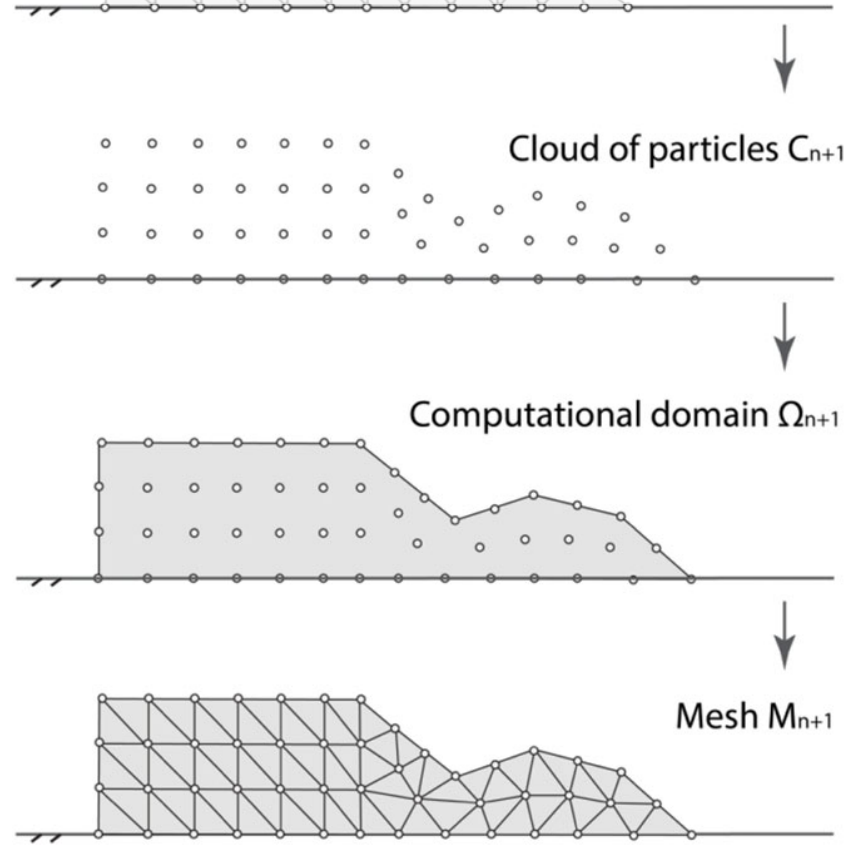

FIGURE 4 Steps for the PFEM modelling of a landslide [Colour figure can be viewed at wileyonlinelibrary.com] 
whether or not the previously solved known states are close to the new unknown states. Such a feature is of great importance in the simulation of landslides, because dramatic changes in the stress or the deformation may occur in a single time interval. More details of the solution algorithm can be found in Zhang et $\mathrm{al}^{26}{ }^{26}$ where the reformulation, the implementation, and the verification have been documented.

\section{RESULTS AND DISCUSSIONS}

\subsection{Analysis using reference parameters}

The problem is first studied using the reference parameters listed in Table 2. Failure of the deposit is triggered by removing the head load, representing an erosion of the slope toe or excavation (Figure 1). The length of the deposit, $100 \mathrm{~m}$, is sufficiently long to remove the boundary effect. A mixed quadratic displacement/linear stress isotropic triangular element $^{26}$ is adopted, and the mesh size is $0.6 \mathrm{~m}(0.066 \mathrm{H})$ leading to a total of 8445 triangular elements and 17248 mesh nodes for the discretisation of the initial computational domain. The simulation proceeds until the final deposit is computed using a time step $\Delta t=0.025$ second.

\subsubsection{Failure mechanism}

The complete failure process of the deposit because of the retreat of the head load is illustrated in Figure 5. As shown, the slope is unstable after the head load is removed. The shear stress at the bottom layer of the slope increases considerably which leads to the yielding of the clay there (Figure 5A). An apparent shear band denoted by C1S1 (eg, the first shear band in the first retrogressive collapse process) emerges, and the plastic strain accumulates at the bottom of the initial slope (Figure 5A). Later, a second shear band, C1S2 (the second shear band in the first retrogressive collapse pro-

cess), emerges as shown in Fi disturbed material migrate in a rigid body, the disturbed clay
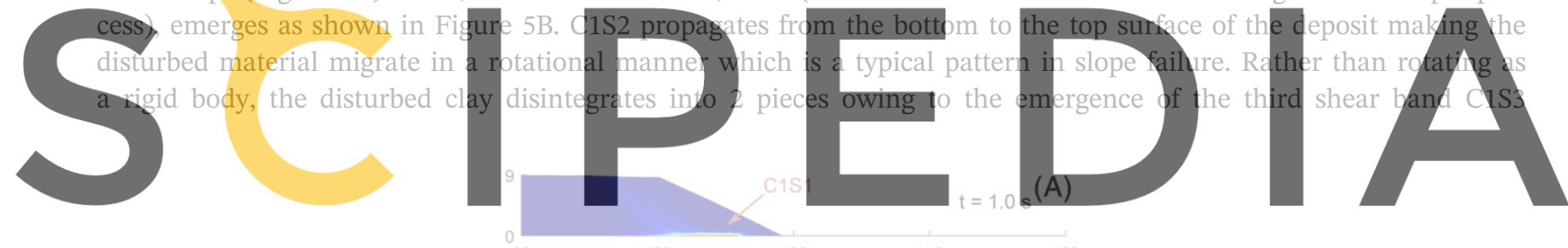

Register for free at https//wwiv.scipedia.com²to download the日eversion without the watermark

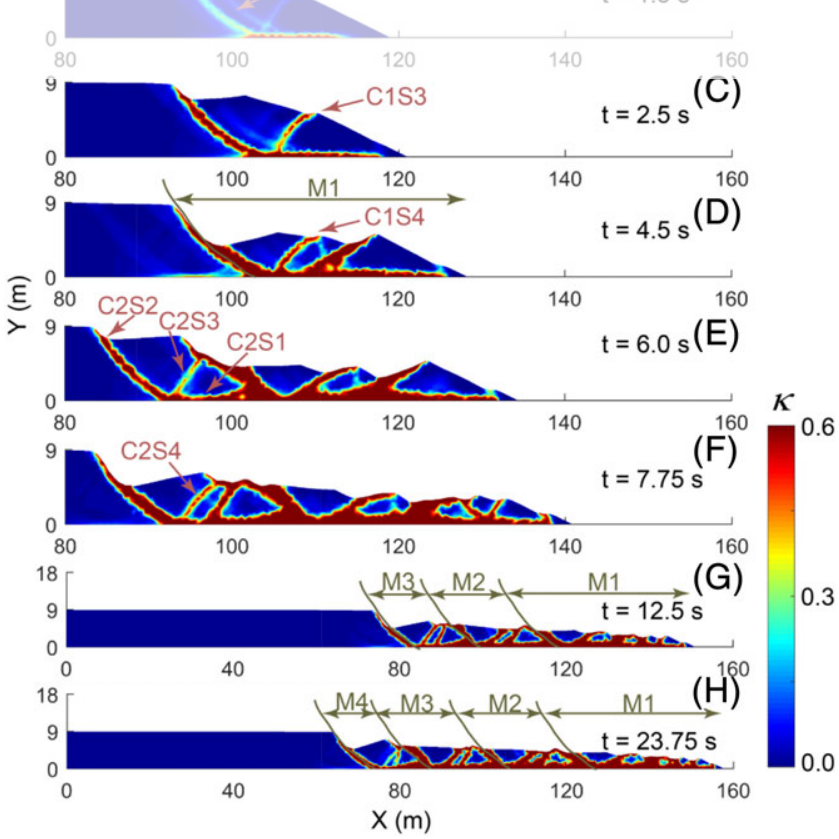

FIGURE 5 Retrogressive failure of the deposit. Colours are proportional to the accumulated equivalent deviatoric plastic strain. Symbol CiSj denotes the $j$ th shear band in the $i$ th retrogressive collapse, and Mi denotes the clay disturbed in the $i$ th retrogressive collapse [Colour figure can be viewed at wileyonlinelibrary.com] 
(Figure 5C) and then further into 3 pieces because of the fourth shear band C1S4 (Figure 5D). Both the shear bands C1S3 and C1S4 originate from the bottom layer of the slope and propagate towards the inclined front surface. Such a failure mechanism of a slope in sensitive clay is termed the multiwedge failure model. ${ }^{44}$

The migration of the clays evoked in the first retrogressive collapse (eg, the mass in the range of M1 as shown in Figure 5D) results in the generation of a new backscarp. This new backscarp is unstable as well (Figure 5E) and fails following the same collapse pattern - the multiwedge failure mode. Four major shear bands (namely C2S1, C2S2, $\mathrm{C} 2 \mathrm{~S} 3$, and C2S4 as shown in Figure 5E, F) emerge sequentially in this retrogressive collapse process and clays involved in this second collapse migrate forwards. Such progressively retrogressive failure continues (Figure 5G) until a stable backscarp is formed (Figure 5H). For the studied problem, the retrogressive failure occurs 4 times (Figure 5H) leading to a considerable amount of materials stored in front of the final stable backscarp. The final run-out distance of the concerned landslide is $40.5 \mathrm{~m}$, and the final retrogression distance is $37 \mathrm{~m}$.

\subsubsection{Kinematics}

Figure 6 shows the variation of the velocity of the sliding front and the maximum velocity of the sliding clay mass in the collapse process. The horizontal velocity of the sliding front increases the moment the head load is removed owing to the transformation of the gravitational potential energy of the disturbed clay into its kinetic energy. After reaching a peak value, the front velocity decreases steadily to zero because of the plastic dissipation and the basal friction. At this reduction stage, the sliding front does not always possess the maximum horizontal velocity. As shown in Figure 6, the horizontal velocity of the sliding front is much lower than the maximum horizontal velocity at $t=10.4$ seconds and $t=14.9$ seconds. On the other hand, the vertical velocity of the sliding front is always zero throughout the failure process, whereas the maximum vertical velocity fluctuates with 4 local maximum values at time instants of 2.9, 6.65,
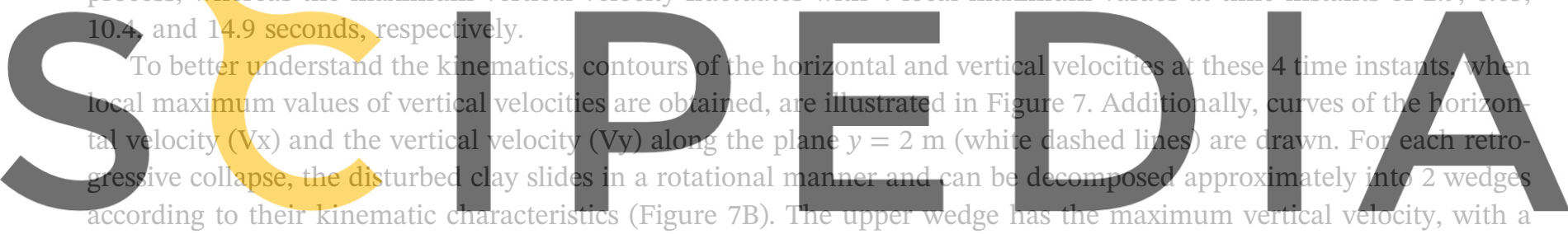

negative sign indicating downward movement caused by the release of the gravitational potential energy, while the

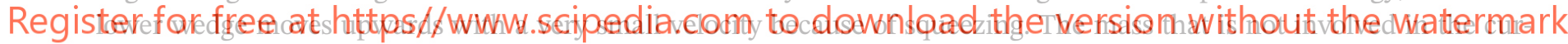
rent collapse moves with a relatively low vertical speed. Thus, the maximum velocity fluctuates whenever a new retrogressive failure is triggered. In contrast, the horizontal velocities of both the upper and lower wedges are positive, indicating that they are moving forward (see Figure 7A). The lower wedge, however, moves much faster than the upper wedge. Unlike the vertical velocity, the horizontal velocity of the sliding front is always considerable in the collapse process. The maximum horizontal velocity during the first 2 collapses (Figure 7(a1) and (a2)) occurs at the sliding front. Although it decreases because of the plastic dissipation and the basal friction, the horizontal velocity of the sliding front

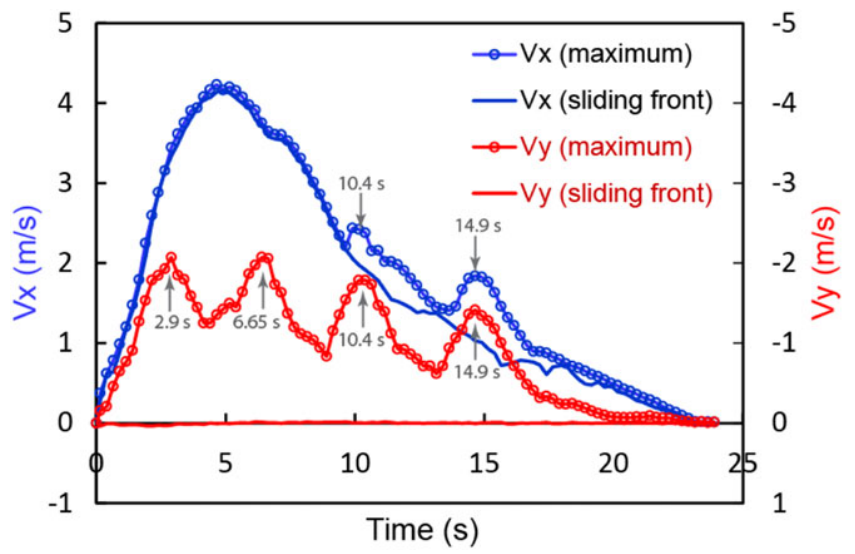

FIGURE 6 Variations of the horizontal and vertical velocities of the sliding front and the maximum horizontal and vertical velocities [Colour figure can be viewed at wileyonlinelibrary.com] 

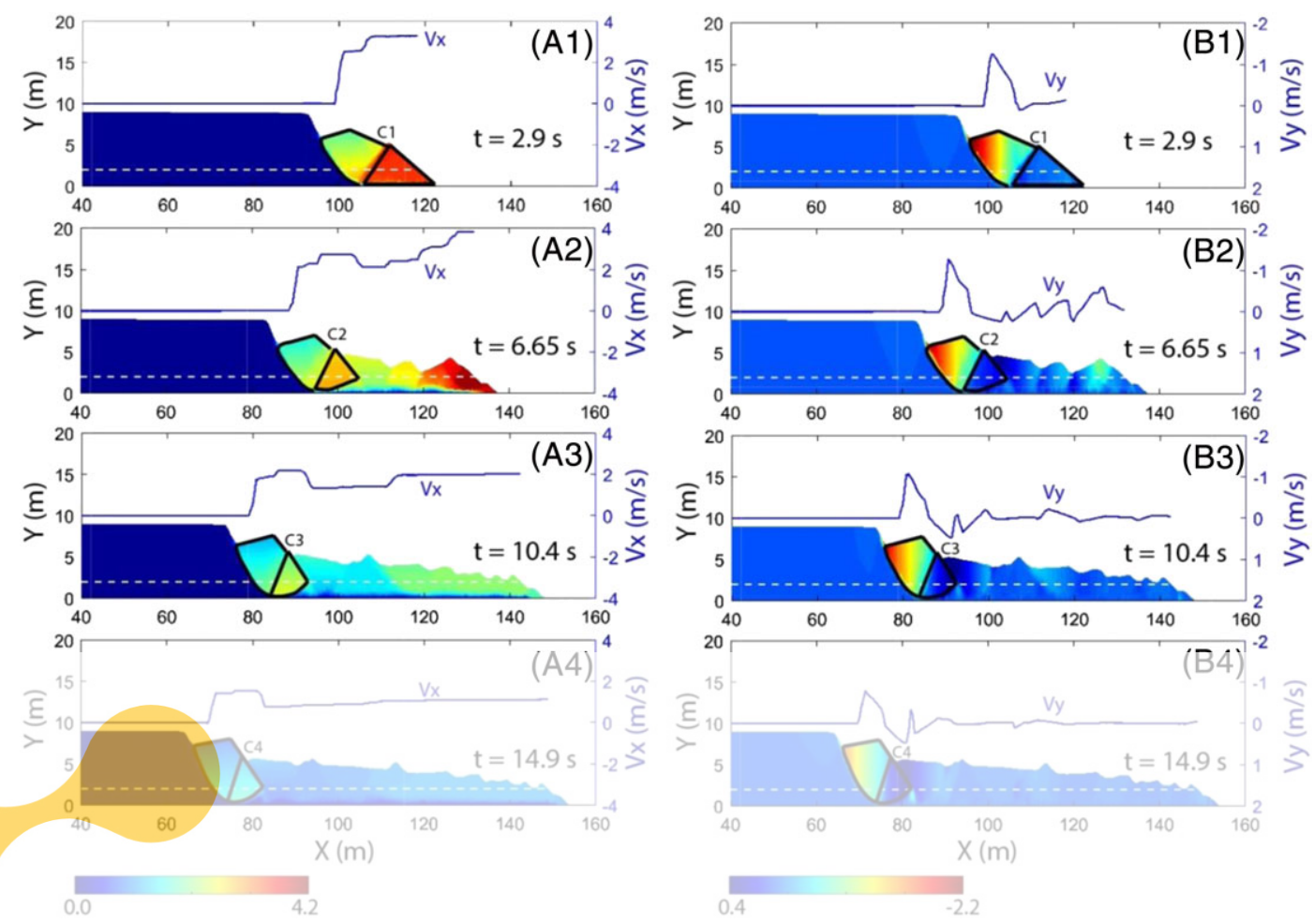

FIGURE 7 Contours of (A) the horizontal velocity, Vx, and (B) the vertical velocity, Vy, at different time instants. Curves correspond to

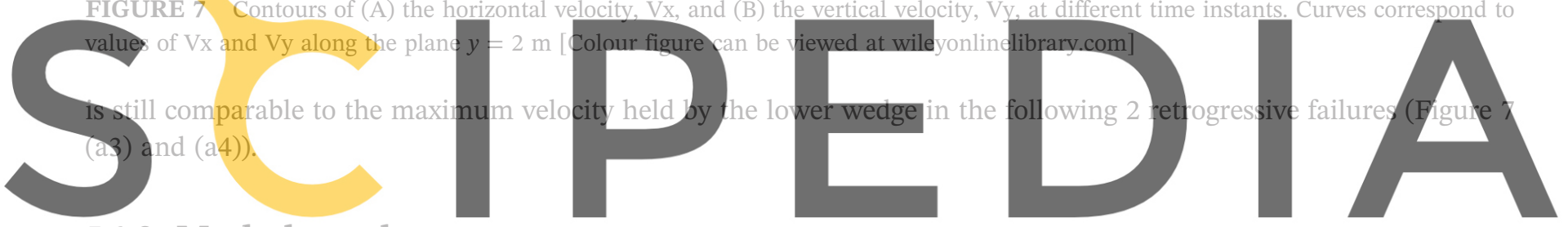

\subsubsection{Mesh dependence}

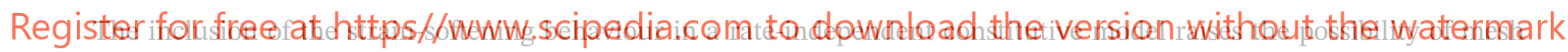
dependence in that the width of a shear band is governed by the mesh size. Fortunately, the elastoviscoplastic model adopted here implicitly introduces a length scale to the corresponding boundary-value problem and thus circumvents this issue. As stated in Moore and Rowe, ${ }^{45}$ however, it is computationally inefficient to capture the small thickness of potential shear bands with a purely continuum model when large-scale practical problems are considered. In this study, we aim to obtain converged solutions regarding the failure patterns and the kinematics of the progressively retrogressive failure rather than to capture the exact width of the shear band which is very thin.

Figure 8 shows the curves of the retrogression distance and the run-out distance against time for the problem modelled using coarse, medium, and fine meshes. The element sizes (the length of the element edge) for these cases are $0.8,0.6$, and $0.4 \mathrm{~m}$, respectively, leading to the total numbers of elements used for discretising the initial domain being 4800 (9875 mesh nodes), 8445 (17248 mesh nodes), and 19116 (38773 mesh nodes). As depicted in Figure 8, converged solutions for both the retrogression distance and the run-out distance are obtained when using the medium and fine meshes. Furthermore, the final depositions obtained from the simulations are compared in Figure 9 where a satisfactory agreement is also achieved. In the following, all simulations were conducted using meshes of a medium size unless otherwise specified.

\subsection{Effect of sensitivity}

To investigate the influence of the clay sensitivity on the retrogressive failure of the deposit, the problem is simulated using St ranging from 1 (for insensitive clay) to above 16 (for quick clay) with the rest of the parameters being the same as listed in Table 2.

As shown in Figure 10, the profile of the final deposition is very dependent of the sensitivity $\mathrm{S}_{\mathrm{t}}$. For the deposit in non-sensitive clays (Figure 10A), the slope fails because of the retreat of the head load and a clear shear band is 

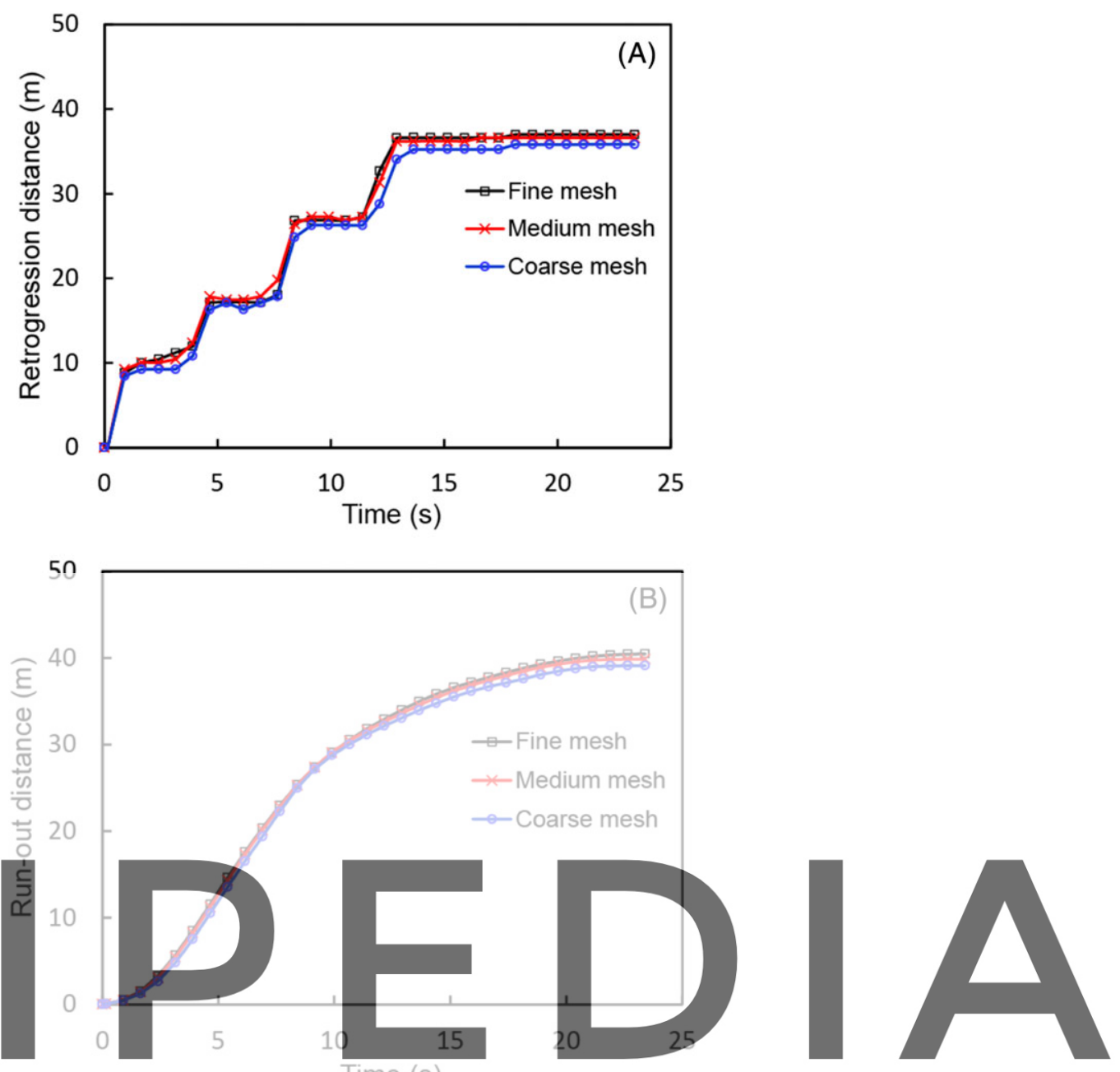

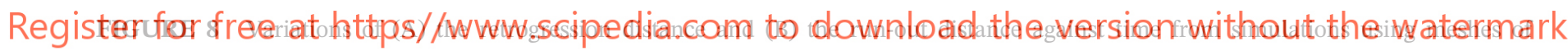
different sizes [Colour figure can be viewed at wileyonlinelibrary.com]

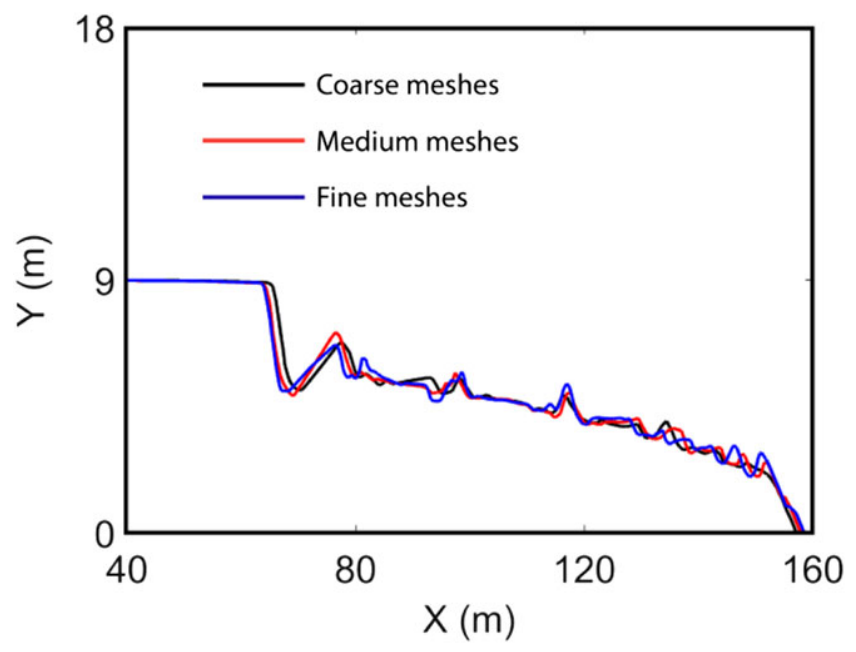

FIGURE 9 Final depositions of the retrogressive failure from simulations using different meshes [Colour figure can be viewed at wileyonlinelibrary.com] 
FIGURE 10 Final depositions of the retrogressive collapse for the deposit in sensitive clays with sensitivity (A) St $=1$, (B) $\mathrm{St}=2$, (C) St $=4$, (D) $\mathrm{St}=8$, and (E) $\mathrm{St}=12$. Colours are proportional to the accumulated equivalent deviatoric plastic strain. $\mathrm{Ci}$ refers to the $i$ th retrogressive collapse [Colour figure can be viewed at wileyonlinelibrary.com]
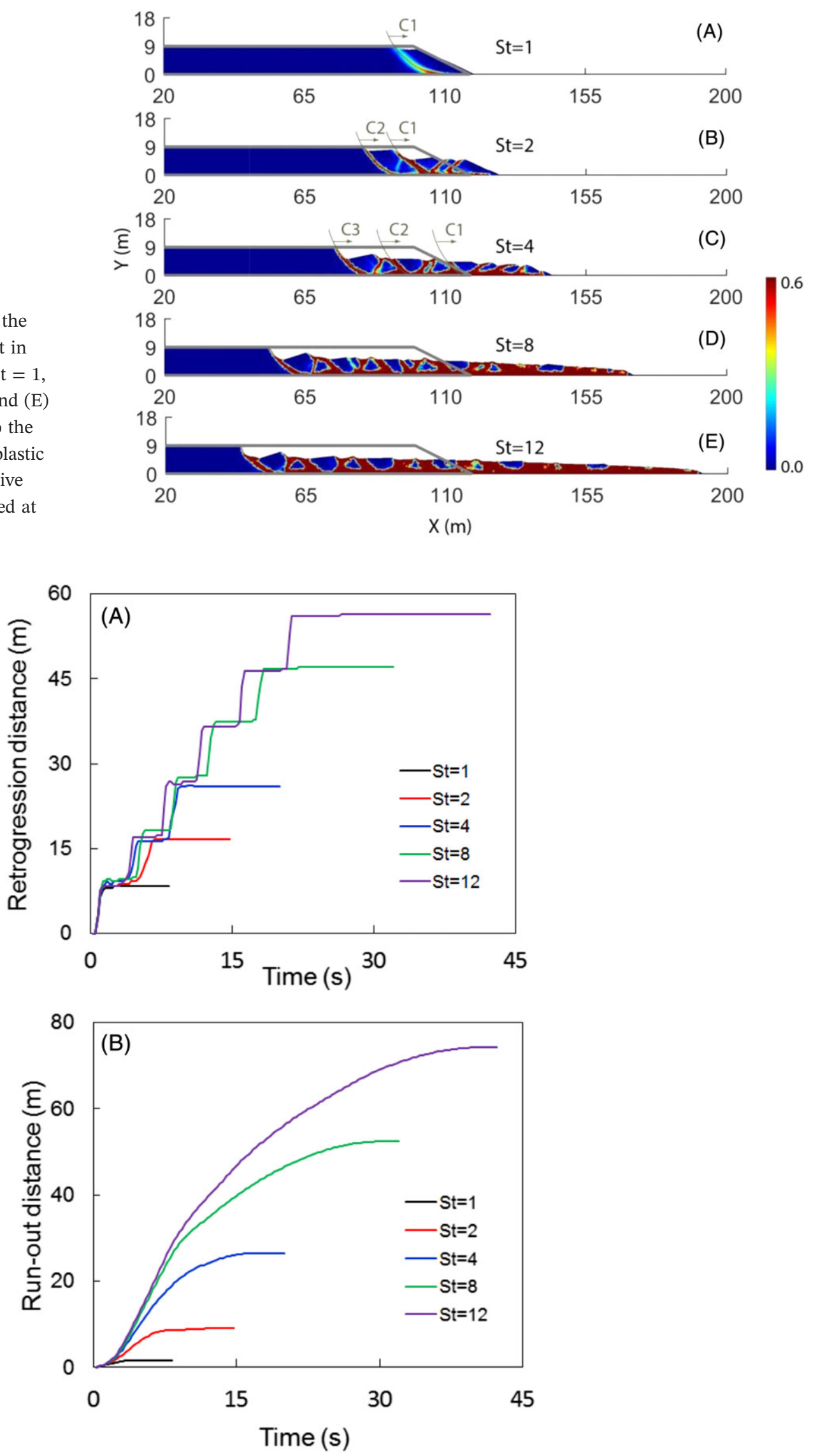

FIGURE 11 Variation of (A) the retrogression distance and (B) the run-out distance of the deposit failure in sensitive clays of different St. [Colour figure can be viewed at wileyonlinelibrary.com] 
observed. Nevertheless, after undergoing very limited deformation the slope becomes stable again. This is because the factor of safety of the slope, estimated using the peak cohesion, is close to 1 and the material has no reduction in strength as the slope deforms. For the deposit in a clay of low sensitivity $\left(S_{t}=2\right)$, the initial slope also fails and the disturbed clay moves away from the newly generated backscarp (Figure 10B). This new backscarp deforms slightly before becoming stable again. A further increase in the sensitivity of the clay leads to more times of the retrogressive failure as shown in Figure 10C to E. A total of 3, 5, and 6 retrogressive failures are observed for the deposit in a medium $\left(S_{t}=4\right)$, highly sensitive $\left(S_{t}=8\right)$, and extra-sensitive $\left(S_{t}=12\right)$ clay as illustrated in Figure 11A where the retrogression distance is plotted against time. This phenomenon can be explained by the fact that same plastic deformation leads to a much lower strength for clays of higher sensitivity. The newly generated backscarp in clays of higher sensitivity is thus more prone to retrogressive failure, and, consequently, a larger retrogression distance will be obtained (Figure 11A). Although the sensitivity influences the times of the retrogressive failure, the increment of the retrogression distance induced by each failure seems to be independent of the sensitivity of clays (Figure 11A). Regarding the run-out distance, Figure 11B indicates that higher sensitivity leads to a larger final run-out distance. Furthermore, it can also be concluded from Figure 11B that the speed of the sliding front (which is the gradient of the curve) is also much larger when the sensitivity is high. Indeed, less energy is dissipated when the clay is fully remoulded because the residual shear strength for higher sensitive clays is much lower. Consequently, a larger proportion of the gravitational potential energy of clays is transformed into their kinematic energy during the collapse that results in a higher speed.

It is also notable that intact clays with a higher sensitivity are more prone to erosion in the sliding process (Figure 10). For the low-sensitive case $(\mathrm{St}=2)$, the block of clays involved in the first retrogressive collapse is divided into 3 undisturbed pieces with remoulded clays existing in between as illustrated in Figure 10B, whereas, for the medium-sensitive case $(\mathrm{St}=4)$, the undisturbed pieces resulting from the first collapse are smaller (Figure 10C). For the highly sensitive and the extra-sensitive cases (eg, St $=8$ and 12), most of the clays involved in the first collapse have been fully remoulded in the sliding process (Figure 10D, E). Moreover, the surface of the final deposition for St $=12$ is much smoother than those for $\mathrm{St}=2$ and 4 . This is owing to the much lower residual shear strength of the extrasensitive clay that it exhibits semifluid behaviour after being fully remoulded.

To further reveal the effect of the strength reduction on the final deposition, plots of the normalised retrogression distance (i.e., the ratio of the retrogression distance to the height of the deposit $H$ ) and the normalised run-out distance (i.e., the ratio of the run-out distance to the height of the deposit $H$ ) as functions of the sensitivity St are shown in Figure 12. As expected, increasing sensitivity leads to an increase of both the retrogression distance and the run-out distance. Owing to the fact that the sensitivity St is a power function rather than a linear function of the remoulded strength, the change rate declines somewhat as St increases. Alternatively, the brittleness of the clay, $\left(c_{\text {up }}-c_{\text {ur }}\right) / c_{\text {up }}$, which is a linear function of the remoulded strength and ranges from 0 to 1 , can be used to characterise the normalised retrogression and run-out distances. ${ }^{46}$ As shown in Figure 13, both the retrogression distance and the run-out distance increase sharply when the brittleness of the clay approaches 1 . In other words, when the remoulded strength is infinitesimal, both the run-out distance and retrogression distance become unbounded.

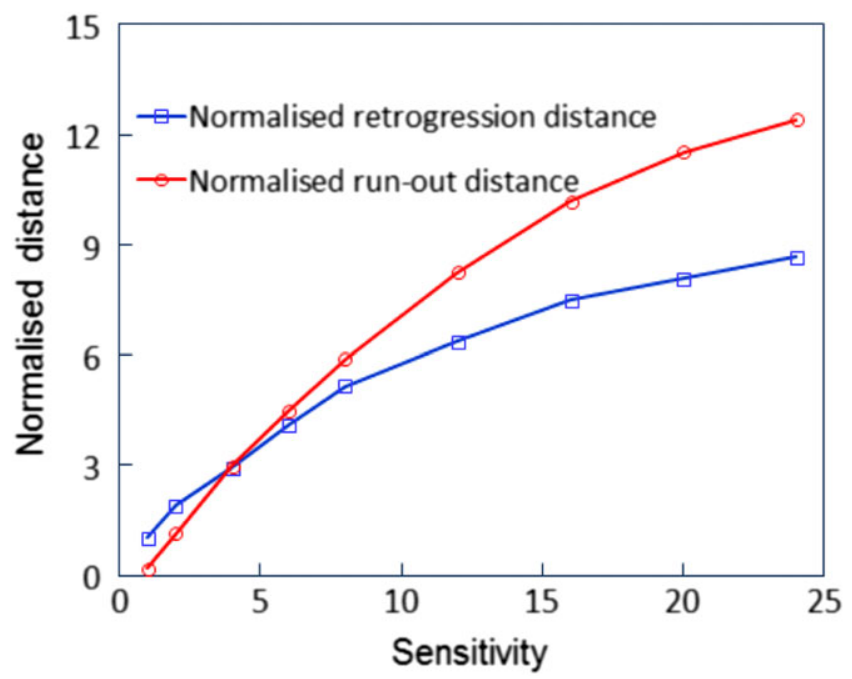

FIGURE 12 Effects of the sensitivity of the clay on the normalised final retrogression distance and the normalised final run-out distance [Colour figure can be viewed at wileyonlinelibrary.com] 


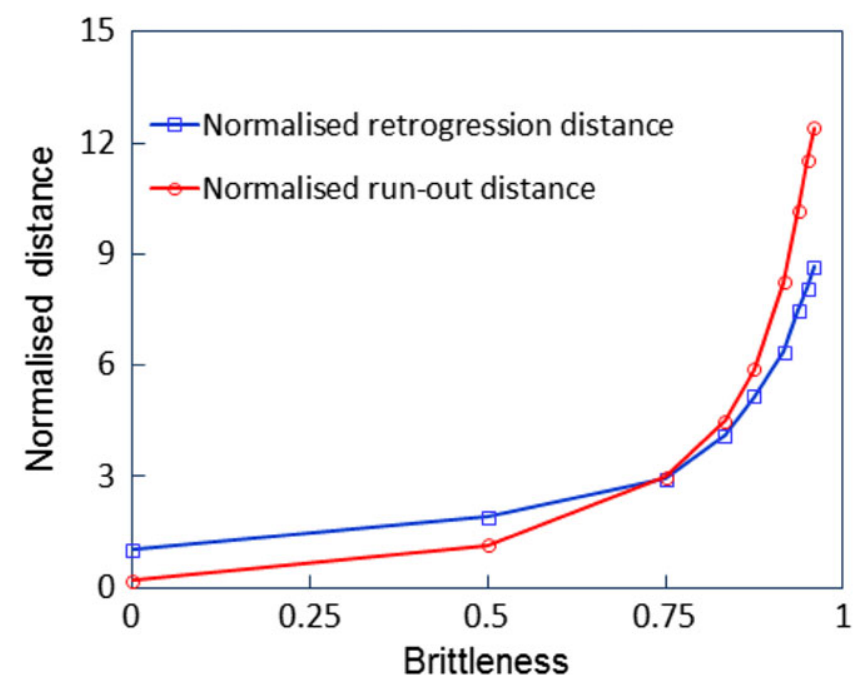

FIGURE 13 Effects of the brittleness of the clay on the normalised final retrogression distance and the normalised final run-out distance [Colour figure can be viewed at wileyonlinelibrary.com]
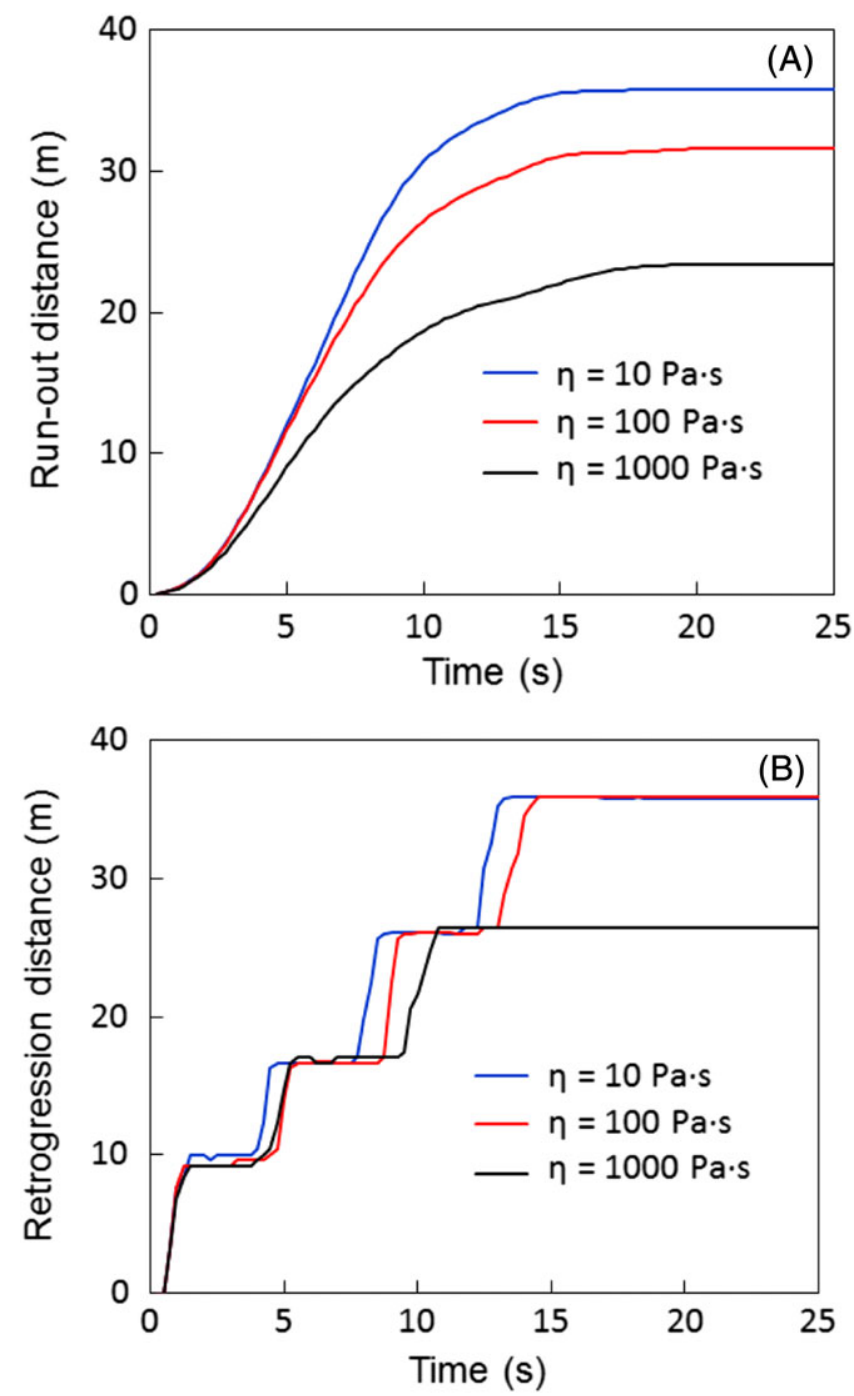

FIGURE 14 Variations of (A) the run-out distance and (B) the retrogression distance for the collapse of the deposit in sensitive clays of different viscosities [Colour figure can be viewed at wileyonlinelibrary.com] 


\subsection{Effect of viscosity}

The effect of viscosity on the retrogressive collapse in a sensitive clay is studied by using different viscosity values ranging from 1 to $1000 \mathrm{~Pa}$ s. This range covers the range of viscosity from both the laboratory tests of a small sample $\mathrm{e}^{31}$ and the back calculation of real-world landslides in sensitive clays. ${ }^{23-25}$ The sensitivity of clays in this case is St $=4$ with other material parameters being the same as those listed in Table 2 .

Plots of the retrogression distance and the run-out distance against time, for various viscosities, are shown in Figure 14. It is clear that an increase in the viscosity leads to a decrease in the final run-out distance (Figure 14A). The final run-out distance for $\eta=10 \mathrm{~Pa} \mathrm{~s}$ is $35.72 \mathrm{~m}$, whereas it is $31.65 \mathrm{~m}$ for $\eta=100 \mathrm{~Pa}$ s and $23.35 \mathrm{~m}$ for $\eta=1000 \mathrm{~Pa} \mathrm{~s}$. This follows from the fact that more energy is dissipated because of viscoplastic deformation when a higher value of viscosity is adopted. In contrast, the relation between the duration of the sliding and the viscosity is not clear from Figure 14A. Indeed, although lower viscosity results in a larger final run-out distance, the leading front also moves at a relatively higher speed. The final retrogression distance is also influenced by the viscosity of the clay. As shown in Figure 14B, 4 times of retrogressive collapse occur for $\eta=10$ and $100 \mathrm{~Pa}$ s resulting in a retrogression distance of $35.85 \mathrm{~m}$, whereas the deposit collapses 3 times for $\eta=1000 \mathrm{~Pa}$ s. Figure 14B also shows that the incremental retrogression distance induced in each collapse is the same regardless of the viscosity. Indeed, the retrogression distance increases when the shear band propagating from the bottom to the top surface (eg, C1S2 and C2S2 in Figure 5) emerges. During this period, the material located in the shear band moves at a very slow speed and the influence of the viscosity is small.

Figure 15 illustrates the final depositions obtained from the simulations using $\eta=10,100$, and $1000 \mathrm{~Pa}$ s. It shows that the failure mode of the retrogressive collapse is independent of the viscosity. Nonetheless, clays are prone to erosion when the viscosity is low. As shown, clays involved in each collapse for $\eta=10 \mathrm{~Pa}$ s disintegrate into much smaller pieces, and more remoulded clays are observed at the front of the deposit when the viscosity is low.

Studies on the influence of the viscosity when the sensitivity of the clay differs are also carried out. Figure 16 shows the final run-out distance and the retrogression distance for $\mathrm{St}=1,2,4$, and 8, respectively. Because the factor of safety for the initial slope is close to 1 , the slope in nonsensitive clay $(\mathrm{St}=1)$ turns out to be stable again after undergoing very limited deformation. In this case, the clay exhibits solid-like behaviour, and thus, neither the runout distance nor the retrogression distance is impacted by the viscosity (Figure 16A). In the case of St $=2$ (lowsensitive clays), the effect of the viscosity on the retrogression distance is still negligible, but the final run-out distance increases from $7.35 \mathrm{~m}$ for high viscosity $\eta=1000 \mathrm{~Pa}$ s up to $11.4 \mathrm{~m}$ for low viscosity $\eta=1 \mathrm{~Pa}$ second (Figure 16B). Not surprisingly, the influence of the viscosity on the retrogression and run-out distances is considerably large for medium and highly sensitive clays. For the medium-sensitive case $(\mathrm{St}=4)$, Figure 16C indicates a $38.46 \%$ (from 26.55 to $36.02 \mathrm{~m}$ ) and a $52.42 \%$ (from 23.35 to $35.58 \mathrm{~m}$ ) increase in the retrogression distance and the run-out distance, respectively, when the viscosity decreases from 1000 to $1 \mathrm{~Pa}$ s. For the high-sensitive case $(\mathrm{St}=8)$, the retrogression distance and the run-out distance experience an $82.78 \%$ (from 35.78 to $65.4 \mathrm{~m}$ ) and a $73.48 \%$ (from 43.81 to $76.0 \mathrm{~m}$ ) increase, respectively, when the viscosity decreases from 1000 to $1 \mathrm{~Pa}$ s according to Figure 16D. This phenomenon again reflects that remoulded clays behave more like a liquid when the sensitivity is high. It is also notable that, when the viscosity $\eta$ is less than $10 \mathrm{~Pa} \mathrm{~s}$, further decrease of viscosity will not result in apparent difference in the final runout distance and the retrogression distance.

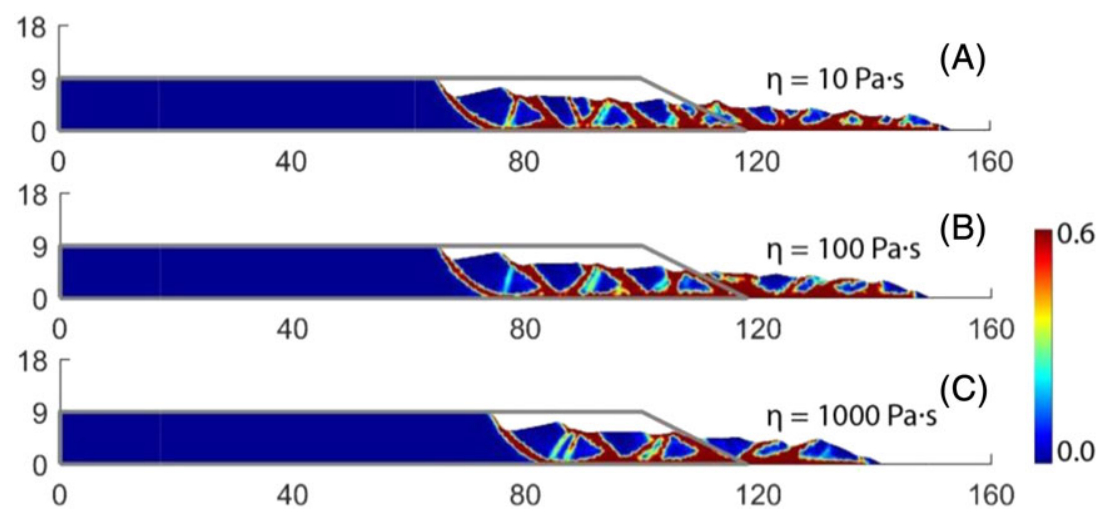

FIGURE 15 Final depositions from the simulations using viscosity (A) $\eta=10$ Pa s, (B) $\eta=100 \mathrm{~Pa} \mathrm{~s}$, and (C) $\eta=1000$ $\mathrm{Pa}$ s. Colours are proportional to the accumulated equivalent deviatoric plastic strain [Colour figure can be viewed at wileyonlinelibrary.com] 

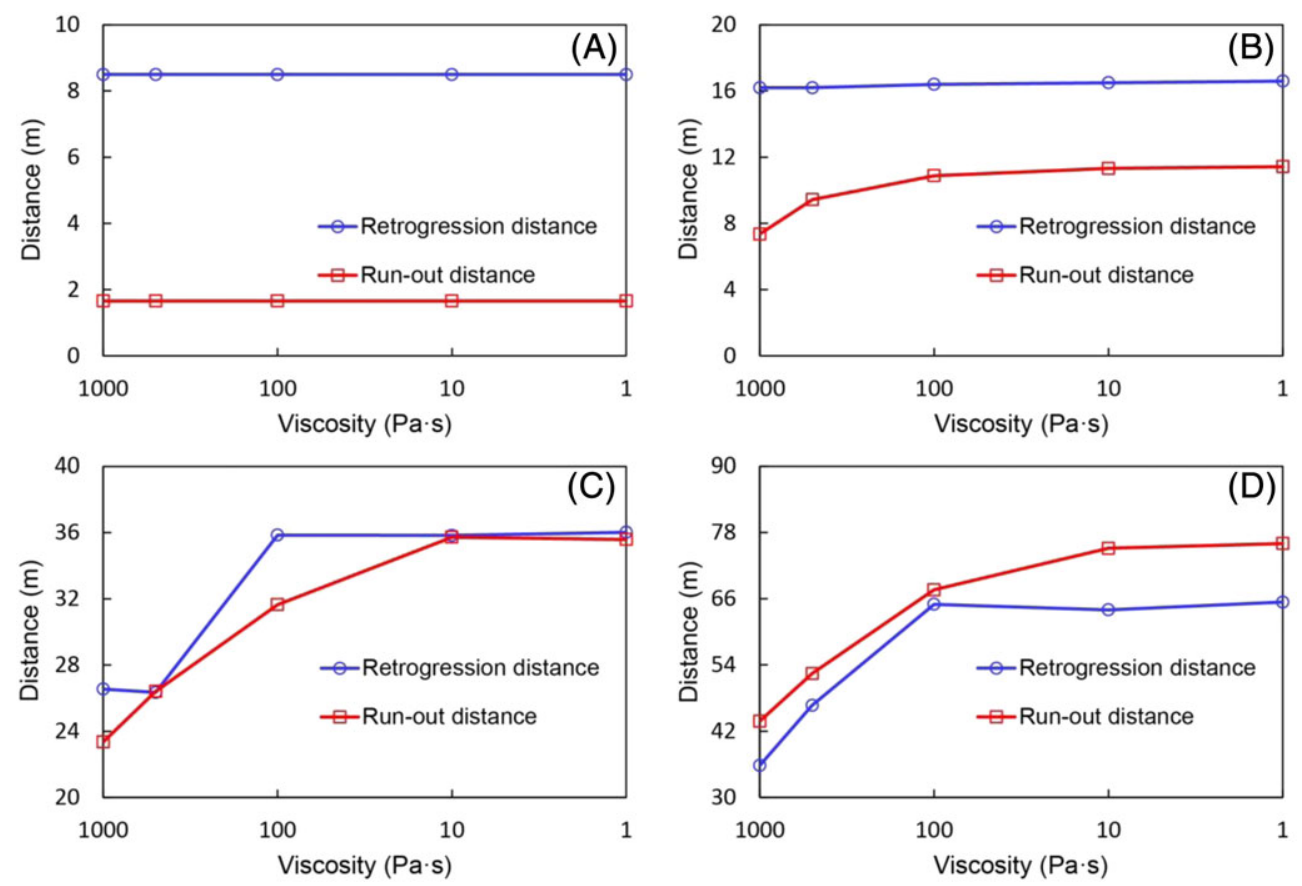

FIGURE 16 Effects of the viscosity on the final retrogression distance and the final run-out distance of the deposit collapse in sensitive clays of (A) St $=1$, (B) St $=2$, (C) St $=4$, and (D) $\mathrm{St}=8$ [Colour figure can be viewed at wileyonlinelibrary.com]

\section{CONCLUSIONS}

In this paper, the retrogressive failure of a deposit in sensitive clay is studied numerically using the PFEM with a strainsoftening elastoviscoplastic model proposed in Zhang et al. ${ }^{26}$ The complete process of multiple retrogressive failures is reproduced successfully.

The multiwedge failure mode composed of 4 main plastic shear bands is reproduced in this study. The complete progressively retrogressive failure of the slope consists of a series of multiwedge failures of new backscarps. In detail, the clay involved in each collapse disintegrates into intact blocks that move like rigid bodies, with the material in the shear bands being fully remoulded and behaving like a fluid. Under continuing deformation, these blocks may decompose further into small pieces during the sliding process. The removal of the clay from the front of a newly generated backscarp may result in further retrogressive collapse. A sequential series of such failures occur until a stable backscarp forms. In spite of the mass in front of the newly generated backscarp, the increment of the retrogression distance induced in each new failure is nearly the same. The speed of the sliding front is always considerable compared to the rest of the slope material throughout the process.

Parametric studies on the influence of the sensitivity and the viscosity of clay have also been conducted. The sensitivity impacts the form of the retrogressive failure significantly. Clays of higher sensitivity are more prone to erosion during the sliding process. An increased sensitivity results in an increase in both the final run-out distance and the retrogression distance. When the remould shear strength $c_{\mathrm{ur}} \rightarrow 0$, both the final run-out distance and the retrogression distance become very large, indicating that unexpected large retrogressive landslides are more likely to occur in sensitive clays of low remoulded strength. For landslides in clays of higher sensitivity, it also takes a longer time for the sliding front to stop completely.

The viscosity of the clay affects the failure. Decreasing the viscosity results in more times of retrogressive failures, and thus increases the corresponding run-out and retrogression distances. Moreover, the intact block of clays resulting from the multiwedge failure is more likely to break up into small fragments when the viscosity is low. However, the viscosity plays a different role in the final run-out distance and the retrogression distance when the clay sensitivity varies. For higher sensitive clays (normally low remoulded shear strength), the influence of the viscosity is more apparent (for instance resulting in a large change in the final run-out distance and the retrogression distance), because, in this case, the remoulded clay behaves more like a liquid. On the other hand, when the sensitivity is low, the clay behaves like a solid even after being fully remoulded in which case the viscosity has a very limited influence. 
Although both the sensitivity and the viscosity of clays considerably impact the number of retrogressive failures, neither of them has considerable influence on the increment of the retrogression distance induced in each new failure.

\section{ACKNOWLEDGEMENTS}

The authors wish to acknowledge the support of European Commission H2020 Marie Skłodowska-Curie actions individual fellowship (Reference 744281) and the Australian Research Council Centre of Excellence for Geotechnical Science and Engineering.

\section{ORCID}

Xue Zhang http://orcid.org/0000-0002-0892-3665

Eugenio Oñate http://orcid.org/0000-0002-0804-7095

\section{REFERENCES}

1. Locat A, Leroueil S, Bernander S, Demers D, Jostad HP, Ouehb L. Progressive failures in eastern Canadian and Scandinavian sensitive clays. Can Geotech J. 2011;48(11):1696-1712.

2. Thakur V, Degago SA. Disintegration energy of sensitive clays. Géotechnique Letters. 2013;3(1):20-25.

3. Zhang W, Wang D, Randolph MF, Puzrin AM. Dynamic propagation criteria for catastrophic failure in planar landslides. Int $J$ Numer Anal Methods Geomech. 2016;40(17):2312-2338.

4. Puzrin AM, Gray TE, Hill AJ. Significance of the actual nonlinear slope geometry for catastrophic failure in submarine landslides. Proc Royal Soc A: Math, Phys Eng Sci. 2015;471(2175). 20140772

5. Quinn PE, Diederichs MS, Rowe RK, Hutchinson DJ. A new model for large landslides in sensitive clay using a fracture mechanics approach. Can Geotech J. 2011;48(8):1151-1162.

6. Lo KY. An approach to the problem of progressive failure. Can Geotech J. 1972;9(4):407-429.

7. Thakur V, Degago SA. Quickness of sensitive clays. Géotechnique Letters. 2012;2(3):87-95.

8. Khaldoun A, Moller P, Fall A, et al. Quick clay and landslides of clayey soils. Phys Rev Lett. 2009;103(18). 188301

9. Bjerrum L, Landva A. Direct simple-shear tests on a Norwegian quick clay. Géotechnique. 1966;16(1):1-20.

10. Park DS, Kutter BL. Static and seismic stability of sensitive clay slopes. Soil Dyn Earthq Eng. 2015;79:118-129.

11. Mitchell RJ, Markell AR. Flowsliding in sensitive soils. Can Geotech J. 1974;11(1):11-31.

12. Carson MA. On the retrogression of landslides in sensitive muddy sediments. Can Geotech J. 1977;14(4):582-602.

13. Mitchell, R. J., Earthflow terrain evaluation in Ontario. 1978.

14. Strand S-A, Thakur V, L'Heureux J-S, et al. Runout of landslides in sensitive clays. In: Thakur V, L'Heureux J-S, Locat A, eds. Landslides in Sensitive Clays: From Research to Implementation. Cham: Springer International Publishing; 2017:289-300.

15. Skempton AW. Long-term stability of clay slopes. Géotechnique. 1964;14(2):77-102.

16. Troncone A. Numerical analysis of a landslide in soils with strain-softening behaviour. Géotechnique. 2005;55(8):585-596.

17. Belytschko T, Lasry D. A study of localization limiters for strain-softening in statics and dynamics. Comput Struct. 1989;33(3):707-715.

18. Wang D, Randolph MF, White DJ. A dynamic large deformation finite element method based on mesh regeneration. Comput Geotech. 2013;54:192-201.

19. Dey R, Hawlader B, Phillips R, Soga K. Large deformation finite-element modelling of progressive failure leading to spread in sensitive clay slopes. Géotechnique. 2015;65(8):657-668.

20. Dey R, Hawlader B, Phillips R, Soga K. Modeling of large-deformation behaviour of marine sensitive clays and its application to submarine slope stability analysis. Can Geotech J. 2016;53(7):1138-1155.

21. Wang B, Vardon PJ, Hicks MA. Investigation of retrogressive and progressive slope failure mechanisms using the material point method. Comput Geotech. 2016;78:88-98.

22. Dey R, Hawlader B, Phillips R, Soga K. Numerical modeling of combined effects of upward and downward propagation of shear bands on stability of slopes with sensitive clay. Int J Numer Anal Methods Geomech. 2016;40(15):2076-2099.

23. Edgers L, Karlsrud K. Soil flows generated by submarine slides-case studies and consequences. Norwegian Goetech Inst, Publ. 1982;143:1-10.

24. Johnson AM, Rodine JR. Debris flow. In: Brunsden D, Prior DB, eds. Slope instability. New York: Wiley; 1984:257-362. 
25. Gauer P, Kvalstad TJ, Forsberg CF, Bryn P, Berg K. The last phase of the Storegga slide: Simulation of retrogressive slide dynamics and comparison with slide-scar morphology. Mar Pet Geol. 2005;22(1):171-178.

26. Zhang X, Sheng D, Sloan SW, Bleyer J. Lagrangian modelling of large deformation induced by progressive failure of sensitive clays with elastoviscoplasticity. Int J Numer Methods Eng. 2017;112(8):963-989.

27. Oñate E, Celigueta MA, Idelsohn SR, Salazar F, Suárez B. Possibilities of the particle finite element method for fluid-soil-structure interaction problems. Comput Mech. 2011;48(3):307-318.

28. Zhang X, Krabbenhoft K, Pedroso DM, et al. Particle finite element analysis of large deformation and granular flow problems. Comput Geotech. 2013;54:133-142.

29. Zhang X, Krabbenhoft K, Sheng D, Li W. Numerical simulation of a flow-like landslide using the particle finite element method. Comput Mech. 2015;55(1):167-177.

30. Llano-Serna MA, Farias MM, Pedroso DM. An assessment of the material point method for modelling large scale run-out processes in landslides. Landslides. 2016;13(5):1057-1066.

31. Locat J, Demers D. Viscosity, yield stress, remolded strength, and liquidity index relationships for sensitive clays. Can Geotech J. 1988;25(4):799-806.

32. Potts DM, Dounias GT, Vaughan PR. Finite element analysis of progressive failure of Carsington embankment. Géotechnique. 1990;40(1):79-101.

33. Skemption AW, Northey RD. The sensitivity of clays. Géotechnique. 1952;3(1):30-53.

34. He P, Ohtsubo M, Higashi T, Kanayama M. Sensitivity of salt-leached clay sediments in the Ariake Bay Area, Japan. Mar Georesour Geotechnol. 2015;33(5):429-436.

35. Yafrate N, DeJong J, DeGroot D, Randolph M. Evaluation of remolded shear strength and sensitivity of soft clay using full-flow penetrometers. J Geotech Geoenviron Eng. 2009;135(9):1179-1189.

36. Oñate E, Celigueta MA, Idelsohn SR, Salazar F, Suárez B. Possibilities of the particle finite element method for fluid-soil-structure interaction problems. Comput Mech. 2011;48(3):307-318.

37. Franci A, Zhang X. 3D numerical simulation of free-surface Bingham fluids interacting with structures using the PFEM. J Non-Newtonian Fluid Mech. 2018;259:1-15.

38. Dávalos C, Cante J, Hernández JA, Oliver J. On the numerical modeling of granular material flows via the particle finite element method (PFEM). Int J Solids Struct. 2015;71:99-125.

39. Zhang X, Krabbenhoft K, Sheng D. Particle finite element analysis of the granular column collapse problem. Granul Matter. 2014;16(4):609-619.

40. Cante J, Dávalos C, Hernández JA, et al. PFEM-based modeling of industrial granular flows. Comput Part Mech. 2014;1(1):47-70.

41. Zhang X, Ding Y, Sheng D, Sloan SW, Huang W. Quasi-static collapse of two-dimensional granular columns: Insight from continuum modelling. Granul Matter. 2016;18(3):1-14.

42. Salazar F, Irazábal J, Larese A, Oñate E. Numerical modelling of landslide-generated waves with the particle finite element method (PFEM) and a non-Newtonian flow model. Int J Numer Anal Methods Geomech. 2016;40(6):809-826.

43. Cremonesi M, Frangi A, Perego U. A Lagrangian finite element approach for the simulation of water-waves induced by landslides. Comput Struct. 2011;89(11-12):1086-1093.

44. Kvalstad TJ, Andresen L, Forsberg CF, Berg K, Bryn P, Wangen M. The Storegga slide: Evaluation of triggering sources and slide mechanics. Mar Pet Geol. 2005;22(1):245-256.

45. Moore ID, Rowe RK. Numerical models for evaluating progressive failure in earth structures-a review. Comput Geotech. 1988;6(3):217-239.

46. Yerro A, Alonso EE, Pinyol NM. Run-out of landslides in brittle soils. Comput Geotech. 2016;80(Supplement C):427-439. 